\title{
Control Design and Stability Analysis of Power Converters: the MIMO Generalized Bode Criterion
}

\author{
Javier Samanes, Member, IEEE, Andoni Urtasun, Member, IEEE, Ernesto Barrios, Member, IEEE, \\ David Lumbreras, Student Member, IEEE, Jesus Lopez, Member, IEEE, Eugenio Gubia, Member, IEEE, \\ and Pablo Sanchis, Senior Member, IEEE
}

\begin{abstract}
Three-phase dynamic systems and multiphase generators are frequently modeled and controlled in the synchronous reference frame. To properly model the cross-coupling terms in this reference frame, complex vector theory and transfer function matrices are commonly applied, obtaining multipleinput multiple output (MIMO) dynamic models. The stability of MIMO systems can be assessed through the Nyquist Generalized Stability Criterion. However, the use of the Nyquist diagram complicates the controller design. The Bode diagram is a more intuitive tool for the controller design, however, the Bode Stability Criterion is not applicable to MIMO systems. In this paper, the MIMO Generalized Bode Criterion is proposed. Since this stability criterion is based on the Nyquist Generalized Stability Criterion it can be applied to any system. Furthermore, it is simple to use, as it only requires information contained in the open-loop transfer matrix and the Bode diagram. The proposed stability criterion thus offers an interesting tool for the controller design procedure in MIMO systems, as it is shown in in this paper for two common applications: the current control loop of a power converter, a $2 \times 2$ system, and the current control loop of two independent power converters in parallel, a $4 \times 4$ system.
\end{abstract}

Index Terms-MIMO systems, stability criterion, Nyquist stability criterion, frequency domain analysis, power converter control.

\section{INTRODUCTION}

$\mathbf{I}$ $\mathrm{N}$ the early stages of power electronics, the synchronous reference frame control, became increasingly popular, as it made it possible to obtain time-independent reluctances in electrical machines while achieving zero tracking error by means of a simple PI controller. At the same time, with the development of renewable energies, many power converters were connected to the grid, adapting the power generated to the grid requirements. These power converters had normally low to medium rated powers, and thus, high switching and sampling frequencies. Consequently, the low delays that appeared in the control loops allowed the cross-coupling terms obtained as a result of $d q$ control to be properly decoupled [1], [2], [3], [4]. Thus, the system could be analyzed as a SISO system [5]. However, in order to reduce converters cost, their rated power has progressively been increased. These high-power converters normally have a reduced switching frequency to limit the power losses, and thus, they have higher delays in the control loop. As a result, the decoupling strategies now become

The authors are with the Department of Electrical, Electronic and Communications Engineering, and the Institute of Smart Cities, Public University of Navarre, 31006, Pamplona, Spain, e-mail: javier.samanes@unavarra.es.

This work has been supported by the Spanish State Research Agency (AEI) and FEDER-UE under grant DPI2016-80641-R. less effective. Although MIMO models take into account $d q$ cross-coupling effects, they present a higher complexity than SISO models. As an alternative, resonant controllers in the stationary reference frame, $\alpha \beta$, are gaining attention [6], [7]. However, the current references are normally computed in the $d q$ axis, so, to properly determine the system stability, a MIMO modeling approach is always required [8], [9].

In order to obtain accurate models of systems controlled in $d q$ coordinates, transfer function matrices, for both symmetric and non-symmetric systems, and complex transfer functions, for symmetric systems, have recently gained attention [10], [11], [12], [13]. In this paper, the scope is focused on transfer function matrices, as they can be used to model any MIMO system. This modeling approach has been applied to three-phase dynamic systems [14], where $2 \times 2$ multiple-input multiple-output (MIMO) systems are obtained as a result of the modeling procedure. This theory has also been used to model multiphase systems, such as in multiple winding generators, where the number of inputs and outputs can be high depending on the number of phases [15], [16]. Transfer function matrices properly model the system dynamics. However, the controller design and the stability analysis become more complicated.

An adequate design of the converter control is extremely important when the number of power converters connected to the grid is increased. Nowadays, power converters are widely used for renewable energy applications, STATCOMS and drives. Their connection to different grids, especially to weak grids, has risen stability issues. The great variations in the grid impedance at the point of connection is forcing to develop robust controllers [17], [18], while the requirements imposed by the grid codes to power converters are creating a need for advanced control designs. Among the issues detected in the existing literature, some of the most recurring ones are the resonance of the output filter of the power converter [19], [17], phase-locked loop instabilities (PLL) [20] and current control loop instabilities [3], [21]. In order to analyze control loop stability, it has been shown that the Bode diagram is a more intuitive diagram compared to Nyquist. For this reason, Bode stability criterion [22], as well as its revised variations [23], have been widely applied to the stability analysis of SISO control loops in power converters. However, these criteria are only valid under certain application conditions and can fail in correctly determining the stability of some systems. Recently, a new criterion, called Generalized Bode Criterion, has been proposed that overcomes these limitations and can be applied to any SISO system [24]. 
MIMO controllers have been designed in power electronics literature using internal model control theory [25], impedance shaping [14], complex vectors [26], [1] and model predictive control [27]. Concerning stability analysis, the dominant approach to assess the system stability in MIMO systems, is the Generalized Nyquist Stability Criterion [28], which is based on the analysis of the open-loop matrix eigenvalues' Nyquist diagram. This criterion is a generalization of the Nyquist criterion from SISO to MIMO systems. However, as it is based on the Nyquist diagram of the eigenvalues, it has a reduced applicability to design controllers on the basis of a desired system response, specially in complicated control loops. Despite the advantages of Bode diagram in the controller design for SISO systems, the limitations of the Bode Stability Criterion when been applied to MIMO systems have prevented its use. In order to extend the benefits of the Bode diagram to design controllers in MIMO systems, this paper proposes a new criterion, called MIMO Generalized Bode Criterion. This criterion is based on the Nyquist Generalized Stability Criterion, and extends the SISO Generalized Bode Criterion to MIMO systems. The proposed stability criterion only requires information contained in the open-loop transfer function matrix and the eigenvalues' Bode diagram. These benefits are illustrated through the design of a typical control loop such as the $d q$ current control of three-phase gridconnected inverters with an $L C L$ filter.

This paper is organized as follows. Section II presents the Nyquist Generalized Stability Criterion for multivariable systems. In Section III, the MIMO Generalized Bode Criterion is proposed for a $n$-order non-symmetric system. Then, in Section IV, the proposed stability criterion is used to design the controller for two different cases. In the first one, a three-phase converter with an $L C L$ output filter, controlled in $d q$ is modeled using transfer matrices, obtaining a $2 \times 2$ system. The stability is analyzed for this application using the proposed stability criterion, proving to be an intuitive approach for the controller design in multivariable systems. In the second case, the MIMO Generalized Bode Criterion is applied for the controller design of two power converters connected in parallel to the grid, resulting in $4 \times 4$ MIMO system. Again, the proposed criterion and design method proves to be an effective tool, obtaining the desired dynamic response. Finally, in Section V, main conclusions are drawn.

\section{MIMO SYSTEMS AND GENERALIZED NYQUIST STABILITY CRITERION}

\section{A. MIMO systems}

A general MIMO feedback system is represented in Fig. 1, where $[G(s)]$ and $[F(s)]$ are $n \times n$ transfer function matrices and $R(s), E(s), Y(s)$ and $M(s)$ are $n$ dimensional vectors. Matrix $[G(s)]$ can thus be expressed as

$$
[G(s)]=\left[\begin{array}{cccc}
G_{11}(s) & G_{12}(s) & \ldots & G_{1 n}(s) \\
G_{21}(s) & G_{22}(s) & \ldots & G_{2 n}(s) \\
\ldots & \ldots & \ldots & \ldots \\
G_{n 1}(s) & G_{n 2}(s) & \ldots & G_{n n}(s)
\end{array}\right]
$$

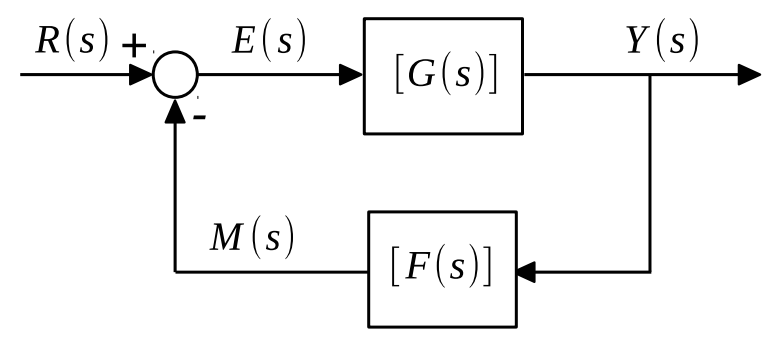

Fig. 1. General closed-loop MIMO system.

and $[F(s)]$ has the same form. The open-loop transfer matrix, $[H(s)]$, for this generic system is given by

$$
M(s)=[H(s)] E(s)=>[H(s)]=[F(s)][G(s)] .
$$

The system closed-loop stability of any MIMO system can be determined from the open-loop transfer matrix by using the Generalized Nyquist Stability Criterion.

\section{B. Generalized Nyquist Stability Criterion}

The Generalized Nyquist stability criterion states that, provided that there are no hidden unstable modes in the system:

the number of closed-loop unstable poles (poles in the right half-plane (RHP)), $Z$, is equal to the number of unstable poles in the open-loop transfer matrix, $P$, minus the total number of anticlockwise encirclements, $N$, around $(-1,0 \mathrm{j})$ of the characteristic loci (Nyquist curves) of all the eigenvalues of $[H(s)][28]$ :

$$
Z=P-N
$$

The eigenvalues of the open-loop transfer matrix, $\lambda$, can be obtained from

$$
|\lambda I-H(s)|=0 .
$$

As a result, unlike a SISO system, the stability is not assessed on the open-loop transfer function, but on the eigenvalues of the open-loop transfer matrix.

Counting the number of open-loop right half plane (RHP) poles in SISO systems is trivial. However, in MIMO systems, the number of unstable poles, $P$, have to be counted for the open loop transfer matrix, $[H(s)]$, expressed in its SmithMcMillan form [28].

An example is used to illustrate the application of the Generalized Nyquist Stability Criterion. A typical MIMO system in power electronics is the case of a three-phase power converter controlled in the synchronous reference frame or $d q$ axis. In any balanced system the homopolar component can be studied independently, so, the open-loop transfer matrix of a three-phase system becomes the $2 \times 2$ matrix

$$
[H(s)]=\left[\begin{array}{ll}
H_{11}(s) & H_{12}(s) \\
H_{21}(s) & H_{22}(s)
\end{array}\right] .
$$

The general expression for its eigenvalues is: 


$$
\begin{aligned}
\lambda_{1,2}(s)= & \frac{H_{11}(s)+H_{22}(s)}{2} \\
& \pm \sqrt{\left(\frac{H_{11}(s)-H_{22}(s)}{2}\right)^{2}+H_{12}(s) H_{21}(s),}
\end{aligned}
$$

and $N$ must be determined by their $(-1,0 \mathrm{j})$ encirclements in the Nyquist diagram.

To determine $P$, which has to be counted in the SmithMcMillan form of $[H(s)]$, lets suppose that $H_{11}(s)=$ $H_{22}(s)=\frac{1}{(s+2)(s-3)}$, while $H_{12}(s)=-H_{21}(s)=\frac{2}{(s-2)}$.

$$
[H(s)]=\left[\begin{array}{cc}
\frac{1}{(s+2)(s-3)} & \frac{2}{(s-2)} \\
-\frac{2}{(s-2)} & \frac{1}{(s+2)(s-3)}
\end{array}\right]
$$

This matrix can be expressed as the product of a $2 \times 2$ polynomial matrix, $[L(s)]$, and a polynomial, $D(s)$, that is equal to the least common multiple of the denominators of all the elements in $[H(s)]$.

$$
[H(s)]=\frac{[L(s)]}{D(s)}
$$

where

$$
D(s)=(s+2)(s-3)(s-2)
$$

and

$$
[L(s)]=\left[\begin{array}{cc}
(s-2) & 2(s+2)(s-3) \\
-2(s+2)(s-3) & (s-2)
\end{array}\right]
$$

From $D(s)$ and $[L(s)]$ the Smith-McMillan form of $[H(s)]$ can be computed. However, to determine the number of unstable poles $P$, only the polynomial $D(s)$ needs to be examined. Consequently, the total number of unstable poles can be computed from (9), obtained as the least common multiple of all the poles in $[H(s)]$. In this example $P=2$.

\section{Proposed STABILITY CRITERION: THE MIMO GENERALIZED BODE CRITERION}

A. Determination of the number of encirclements, $N$, and fundamentals of the criterion

The MIMO Generalized Bode Criterion (MIMO-GBC) translates the stability criterion presented in the previous section to the Bode diagram, so that the controller design is facilitated. $P$ is determined in the MIMO-GBC as already explained, so in the following, the determination of $N$ through the Bode diagram is comprehensively covered.

Counting the total number of encirclements of $(-1,0 \mathrm{j})$ using the Nyquist diagram of the eigenvalues becomes complicated when the order of the system is increased. A simpler approach to compute $N$ was proposed in [29]. An auxiliary ray can be traced in a random direction starting at $(-1,0 \mathrm{j})$, and the crossings of the Nyquist diagram with this ray can be counted to determine $N$. The Nyquist stability criterion can be rewritten as

$$
Z=P-\left(N_{c}^{+}-N_{c}^{-}\right)
$$

where $N_{c}^{+}$are the number of crossings between the Nyquist diagram and the ray when the phase is increasing and $N_{c}^{-}$ are the number of crossings when the phase is decreasing. The positive angle is defined in the anticlockwise direction with respect to the positive real axis of the Nyquist plot. It must be highlighted that in a MIMO system, $N_{c}^{+}$and $N_{c}^{-}$ should be counted for each eigenvalue and added together to compute the total number of encirclements. As the ray can be chosen in any random direction, [29] proposed to draw this ray starting at $(-1,0 \mathrm{j})$ in the direction of the real negative halfaxis. In this way, $N_{c}^{+}$and $N_{c}^{-}$can be counted on the Bode diagram by counting the number of crossings in the phase plot with $\pm m 180$ degrees, $m$ being an odd integer, when the magnitude plot is greater than $0 \mathrm{~dB}$, as represented in Fig. 2 . These crossings counted on the Bode diagram are denoted as $C^{+}$when the phase is increasing and $C^{-}$when the phase is decreasing.

The Nyquist diagram is represented for positive and negative frequencies by substitution of $s$ by $j \omega$, with $-\infty<\omega<\infty$. The Bode diagram is only represented for positive frequencies, $0<\omega<\infty$. This is not a problem in SISO systems, whose coefficients are always real valued. If $G(s)$ is a transfer function with real valued coefficients, it satisfies that $G(j \omega)=$ $\overline{G(-j \omega)}$, meaning that the Bode diagrams are symmetric with respect to zero. Consequently, $C^{+}$and $C^{-}$can be counted for positive frequencies and multiplied by 2 [24].

In MIMO systems, the crossings must be counted for all the eigenvalues, $\lambda$, which might have, in general, complex coefficients. As a result, the system eigenvalues will not be symmetric with respect to $0 \mathrm{~Hz}$. However, the magnitude and phase at negative frequencies of the set of eigenvalues are unambiguously defined by the magnitude and phase of the set of eigenvalues at positive frequencies. Appendix A demonstrates this concept for any two-input two-output MIMO system. It proves that, depending on the system under analysis,

$$
\lambda_{1}(j \omega)=\overline{\lambda_{1}(-j \omega)} \text { and } \quad \lambda_{2}(j \omega)=\overline{\lambda_{2}(-j \omega)}
$$

or

$$
\lambda_{1}(j \omega)=\overline{\lambda_{2}(-j \omega)} \text { and } \quad \lambda_{2}(j \omega)=\overline{\lambda_{1}(-j \omega)} .
$$
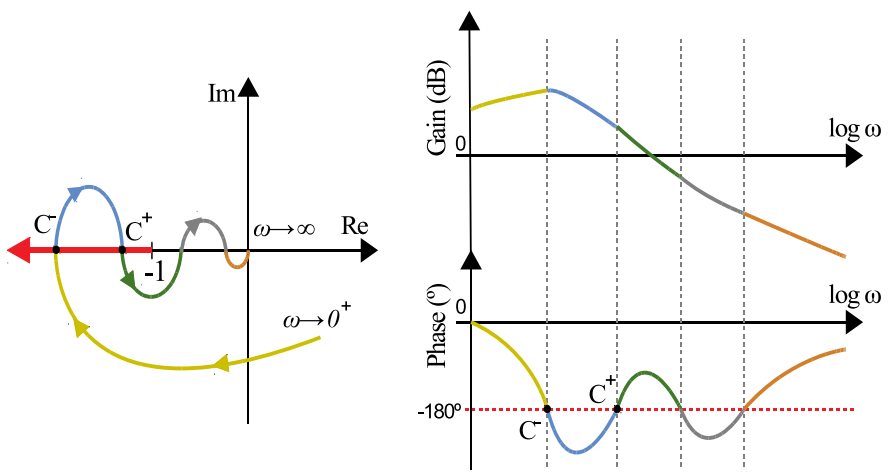

Fig. 2. Equivalence between the Nyquist and Bode diagrams. 
Similarly, although it is not demonstrated, the same behavior is shown for an $m \times m$ system in [28]. In this way, we can count $\pm m 180$ degrees crossings with positive magnitude ( $m$ odd integer) only at positive frequencies in the Bode diagram of all the system eigenvalues, determining the total number of $C^{+}$and $C^{-}$crossings, and multiply the result by 2 to obtain $N$, as in a SISO system.

Besides the crossings $C^{+}$and $C^{-}$identified in the Bode diagram at positive frequencies, additional crossings can occur at $0 \mathrm{~Hz}$, which cannot be seen in the Bode diagram and are key to determine the stability. These crossings are denoted as $C_{0}$, defined as the total number of crossings with $\pm m 180$ degrees ( $m$ odd integer) at $0 \mathrm{~Hz}$ with positive magnitude of the set of eigenvalues. $C_{0}$ is positive if the phase is increasing and negative if it is decreasing. This parameter will be determined in the next Subsection III-B.

Taking everything into account, the MIMO Generalized Bode Criterion can be expressed as

$$
Z=P-\left[2\left(C^{+}-C^{-}\right)+C_{0}\right] .
$$

The condition that any closed-loop system has to meet to be asymptotically stable $(Z=0)$, can thus be expressed as

$$
P=\left[2\left(C^{+}-C^{-}\right)+C_{0}\right] .
$$

\section{B. $C_{0}$ calculation}

The last parameter required to assess the system stability is $C_{0}$. This parameter accounts for the eigenvalues' crossings with $\pm m 180$ degrees that may occur at $0 \mathrm{~Hz}$ and that cannot be counted in the Bode diagram. Again, $C_{0}$ has to be determined for each eigenvalue and added together in order to obtain the number of unstable closed-loop poles in (14).

The general expression of an eigenvalue, $\lambda_{i}(s)$ is

$$
\lambda_{i}(s)=K_{G} \frac{\prod_{q=1}^{N_{z}}\left(\tau_{z, q} s+1\right)}{s^{k} \prod_{q=1}^{N_{p}}\left(\tau_{p, q} s+1\right)}, i=1,2, \ldots, n
$$

where $K_{G}=a+b j, \tau_{z, i}$ and $\tau_{p, i}$ are complex numbers, $N_{z}$ is the number of zeros, $N_{p}$ the number of poles, and $k$ the number of integrators. Each $\lambda_{i}(s)$ is considered to be strictly proper, $N_{p}+k>N_{z}$, as any real system meets this condition [30]. As it will be shown in the following, both $k$ and $K_{G}$, which is the eigenvalue DC gain without integrators, are key parameters to determine $C_{0}$.

The value of $C_{0}$ will be obtained for a maximum number of 2 integrators in the eigenvalues, since a higher number is not of practical application. Nevertheless, the cases for a greater number of integrators are a repetition of the ones studied here. Table I summarizes the possible $C_{0}$ values obtained by analyzing the Nyquist diagrams shown in Fig. 3.

1) Derivatives in the eigenvalue $\lambda_{i}(s), k<0$ : If $k<0$ $\lambda_{i}$ has $k$ derivatives. In this case, there are no possible $C_{0}$ crossings, as the eigenvalue evaluated at $0 \mathrm{~Hz}$ are always zero.
2) No integrators in the eigenvalue $\lambda_{i}(s), k=0$ : In this case, $\lambda_{i}$ evaluated at $0 \mathrm{~Hz}$, is equal to

$$
\lambda_{i}(0)=K_{G}=a+b j .
$$

Consequently, the parameter $C_{0}$ depends on $K_{G}$, as represented by Fig. 3 (a)-(d). If $K_{G}$ has a non-zero imaginary term, $C_{0}$ is equal to 0 , as there are no possible crossings with the ray starting at $(-1,0 \mathrm{j})$ in the direction of the negative real axis (Fig. 3 (a)). If $K_{G}$ is real valued, but greater than -1 , a $C_{0}$ cannot be produced (Fig. 3 (b)). However, if $K_{G}$ is real valued, with $a<-1$, the sign of the positive crossing depends on the phase at $0^{+} \mathrm{Hz}$. Whenever the eigenvalues' phase at a frequency slightly greater than $0 \mathrm{~Hz}$ is lower than $-180^{\circ}, \varphi\left(\lambda_{i}\left(0^{+}\right)\right)<-180^{\circ}$, the eigenvalue Nyquist diagram from $0^{-}$to $0^{+} \mathrm{Hz}$ produces a negative crossing (clockwise), consequently $C_{0}$ is equal to -1 (Fig. 3 (c)). This crossing can be guaranteed because the eigenvalue phase derivative exists an is thus equal at $0^{-}$and $0^{+} \mathrm{Hz}$. This means that, in this case, since the phase decreases at $0^{+}$, it also decreases at $0^{-}$. In general, the eigenvalue's phase derivative is

$$
\left.\frac{d \varphi\left(\lambda_{i}(j \omega)\right)}{d \omega}\right|_{\omega=0}=\sum_{q=1}^{N_{z}} \operatorname{Re}\left(\tau_{z, q}\right)-\sum_{q=1}^{N_{p}} \operatorname{Re}\left(\tau_{p, q}\right) .
$$

On the other hand, if $\varphi\left(\lambda_{i}\left(0^{+}\right)\right)>-180^{\circ}$, a positive crossing (anticlockwise) is produced and thus $C_{0}$ is equal to +1 , as it can be seen in Fig. 3 (d). Again, the symmetry for small frequency variations around $0 \mathrm{~Hz}$ guarantees this crossing.

3) One integrator in the eigenvalue $\lambda_{i}(s), k=1$ : There cannot be poles and zeros on the Nyquist contour, $\Gamma$, so it has to be modified in the presence of integrators. This contour, represented in Fig. 4 is called indented contour, which is defined by

$$
\Gamma:\left\{\begin{array}{l}
1 . j \omega, \varepsilon<\omega<r \\
2 \cdot r e^{j \theta}, \frac{\pi}{2} \geq \theta \geq-\frac{\pi}{2} \\
3 . j \omega,-r<\omega<-\varepsilon \\
A B \cdot \varepsilon e^{j \phi},-\frac{\pi}{2} \leq \phi \leq \frac{\pi}{2}
\end{array}\right.
$$

for $r$ positive and sufficiently large and $\varepsilon$ positive and infinitesimally small. The Nyquist plot is obtained by plotting $\lambda_{i}(\Gamma)$. To calculate $C_{0}$, the expression $\lambda_{i}$ given in (16) with $k=1$, can be evaluated for the semicircle $\mathrm{AB}$

$$
\lambda_{i}\left(\varepsilon e^{j \phi}\right)=\frac{K_{G}}{\varepsilon e^{j \phi}} .
$$

From the previous equation it can be concluded that as $\phi$ changes from $-\pi / 2$ to $\pi / 2$, an integrator introduces $180^{\circ}$ rotation through infinity. This rotation is in the clockwise direction, as it can be deduced if $\lambda_{i}\left(\varepsilon e^{j \phi}\right)$ is evaluated at $-\pi / 2,0$ and $\pi / 2$, obtaining

$$
\begin{gathered}
\lambda_{i}\left(\varepsilon e^{-j \pi / 2}\right)=\lambda_{i}\left(0^{-}\right)=\frac{K_{G}}{\varepsilon} j=\frac{a j-b}{\varepsilon}, \\
\lambda_{i}\left(\varepsilon e^{0}\right)=\frac{K_{G}}{\varepsilon}=\frac{a+b j}{\varepsilon},
\end{gathered}
$$




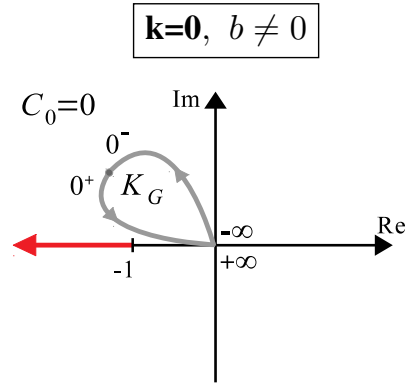

(a)

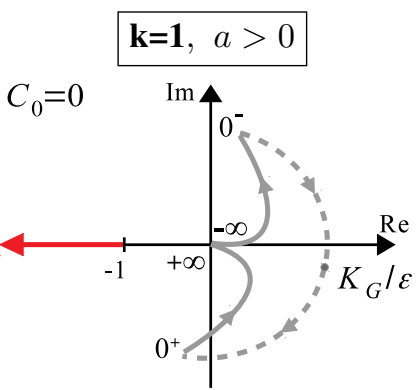

(e)

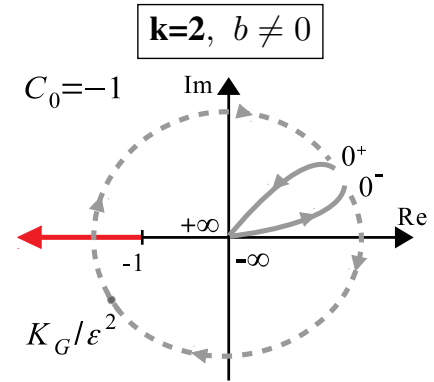

(i) $\mathbf{k}=\mathbf{0}, a>-1$ and $b=0$

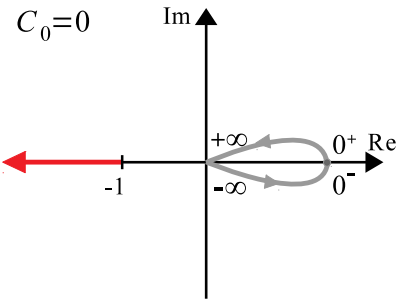

(b)

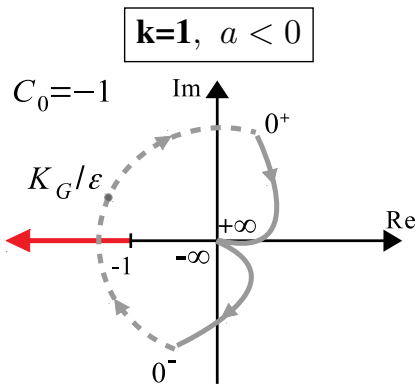

(f)

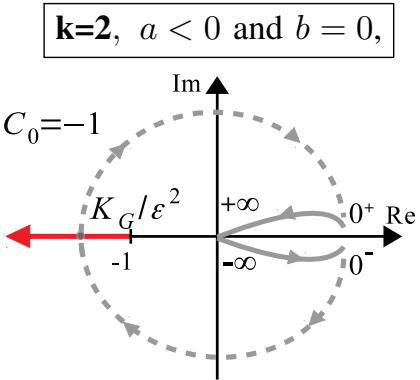

(j)

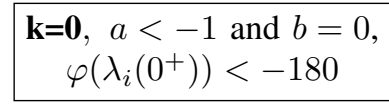

$\mathbf{k}=\mathbf{0}, a<-1$ and $b=0$
$\varphi\left(\lambda_{i}\left(0^{+}\right)\right)>-180$

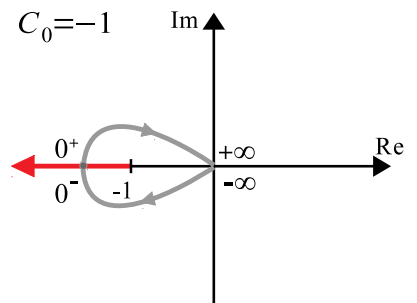

(c)

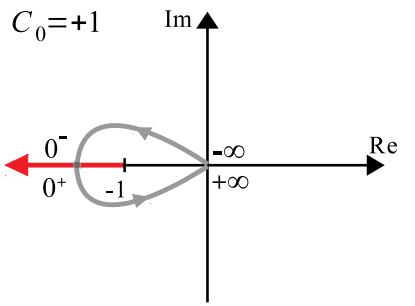

(d)

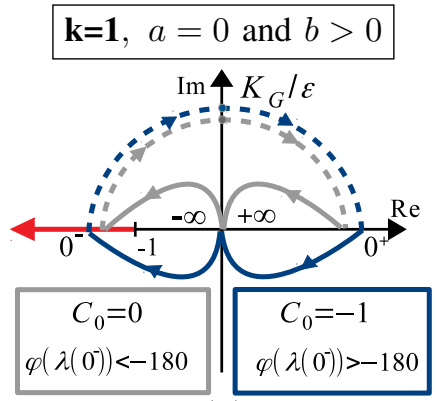

(g)

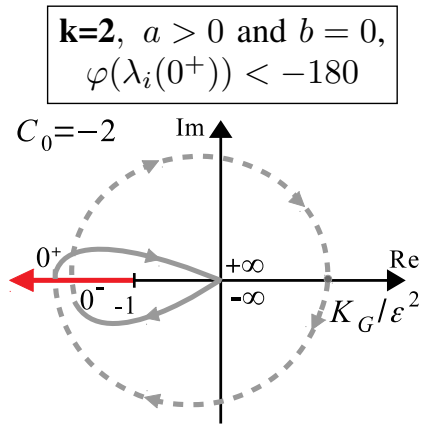

(k)

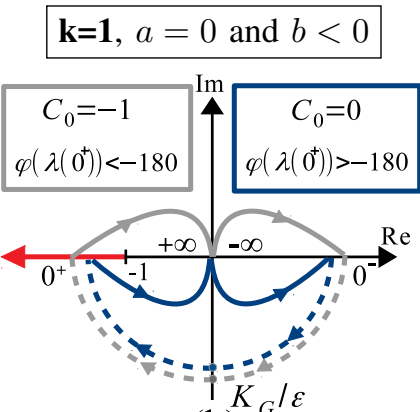

(h)

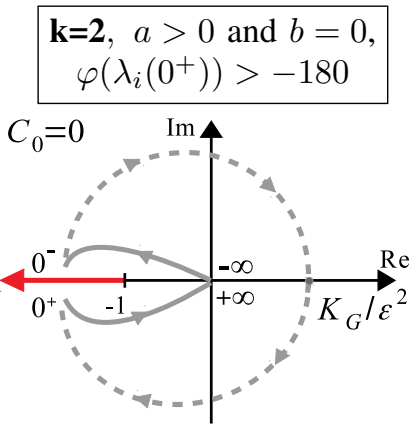

(1)

Fig. 3. Nyquist diagrams to calculate $C_{0}$ depending on the number of integrators and the DC gain without integrators.

$$
\lambda_{i}\left(\varepsilon e^{j \pi / 2}\right)=\lambda_{i}\left(0^{+}\right)=-\frac{K_{G}}{\varepsilon} j=\frac{-a j+b}{\varepsilon}
$$

As represented in Fig. 4, it should also be noted that $\phi=$ $-\pi / 2$ corresponds to frequency $0^{-} \mathrm{Hz}$, while $\phi=\pi / 2$ to

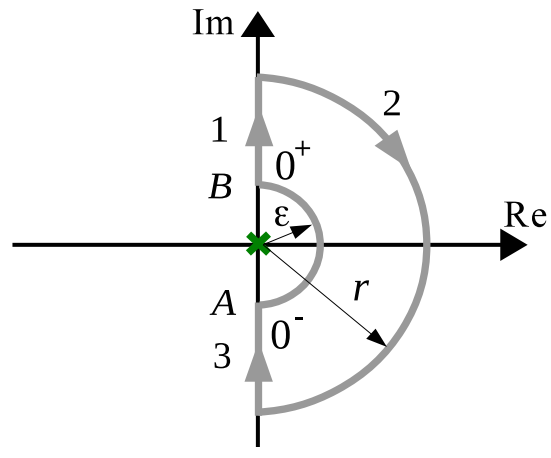

Fig. 4. Indented Nyquist contour. frequency $0^{+} \mathrm{Hz}$. Considering (21)-(23), $C_{0}$ can be computed depending on $K_{G}=a+b j$. If $a>0$ (Fig. 3 (e)), $C_{0}=0$, while if is lower than $0, C_{0}=-1$ (Fig. 3 (f)). If $a=0$, the Nyquist diagram at $0^{+}$and $0^{-} \mathrm{Hz}$ is located in the real axis, so 4 cases must be distinguished under these circumstances. If $b>0$, the phase at $0^{-}$determines the number of crossings, as it can be seen in Fig. $3(\mathrm{~g})$. With $\varphi\left(\lambda_{i}\left(0^{-}\right)\right)<-180^{\circ}, C_{0}$ is equal to 0 , while if it is greater than -180 degrees, a crossing occurs, $C_{0}=-1$. In this paper, the stability is determined using the eigenvalue's response at positive frequencies. It has been proven in (18), that at $0^{+}$and $0^{-}$the eigenvalue has the same phase slope, as the derivative is the same. For this reason, the condition required for a $C_{0}$ crossing in case of $a=0$ and $b>0$ can be expressed by the phase at $0^{+}$. Specifically, if $\varphi\left(\lambda_{i}\left(0^{+}\right)\right)<0, C_{0}=-1$, while if $\varphi\left(\lambda_{i}\left(0^{+}\right)\right)>0, C_{0}=0$.

If $a=0$ and $b<0,0^{+}$falls in the real negative axis and, consequently, $\varphi\left(\lambda_{i}\left(0^{+}\right)\right)$has to be examined. As shown in Fig. $3(\mathrm{~h})$ if $\varphi\left(\lambda_{i}\left(0^{+}\right)\right)<-180^{\circ}, C_{0}=-1$, while no crossings at $0 \mathrm{~Hz}$ are obtained if $\varphi\left(\lambda_{i}\left(0^{+}\right)\right)>-180^{\circ}$. All these cases are summarized in Table I. 
TABLE I

PARAMETER $C_{0}$ FOR EIGENVALUES WITH UP TO TWO INTEGRATORS.

\begin{tabular}{|c|c|c|c|}
\hline $\begin{array}{c}\text { Number of } \\
\text { integrators } \\
\quad(k)\end{array}$ & $\begin{array}{c}\text { DC gain without } \\
\text { integrators } \\
\left(K_{G}=a+b j\right) \\
\end{array}$ & $\begin{array}{c}\text { Phase at } \\
0^{+} \text {and } 0^{+} \mathrm{Hz} \\
\varphi\left(\lambda_{i}\left(0^{+}\right)\right) \text {and } \varphi\left(\lambda_{i}\left(0^{-}\right)\right)\end{array}$ & $\begin{array}{c}\text { Crossings } \\
\text { at } 0 \mathrm{~Hz} \\
C_{0}\end{array}$ \\
\hline$k<0$ & - & - & 0 \\
\hline \multirow{4}{*}{$k=0$} & $b \neq 0$ & - & 0 \\
\hline & $a>-1, b=0$ & - & 0 \\
\hline & \multirow{2}{*}{$a<-1, b=0$} & $\varphi\left(\lambda_{i}\left(0^{+}\right)\right)>-180$ & +1 \\
\hline & & $\varphi\left(\lambda_{i}\left(0^{+}\right)\right)<-180$ & -1 \\
\hline \multirow{6}{*}{$k=1$} & $a<0$ & - & -1 \\
\hline & $a>0$ & - & 0 \\
\hline & \multirow{2}{*}{$a=0, b>0$} & $\varphi\left(\lambda_{i}\left(0^{+}\right)\right)<0$ & -1 \\
\hline & & $\varphi\left(\lambda_{i}\left(0^{+}\right)\right)>0$ & 0 \\
\hline & \multirow{2}{*}{$a=0, b<0$} & $\varphi\left(\lambda_{i}\left(0^{+}\right)\right)>-180$ & 0 \\
\hline & & $\varphi\left(\lambda_{i}\left(0^{+}\right)\right)<-180$ & -1 \\
\hline \multirow{4}{*}{$k=2$} & $b \neq 0$ & - & -1 \\
\hline & $b=0, a<0$ & - & -1 \\
\hline & \multirow{2}{*}{$b=0, a>0$} & $\varphi\left(\lambda_{i}\left(0^{+}\right)\right)>-180$ & 0 \\
\hline & & $\varphi\left(\lambda_{i}\left(0^{+}\right)\right)<-180$ & -2 \\
\hline
\end{tabular}

4) Two integrators in the eigenvalue $\lambda_{i}(s), k=2$ : The same method is followed for two integrators. In this case, the eigenvalue evaluated in $\mathrm{AB}$ is

$$
\lambda_{i}\left(\varepsilon e^{j \phi}\right)=\frac{K_{G}}{\varepsilon^{2} e^{j 2 \phi}} .
$$

There are 2 integrators, so a 360 degree clockwise revolution in the Nyquist diagram is obtained:

$$
\begin{gathered}
\lambda_{i}\left(\varepsilon e^{-j \pi / 2}\right)=-\frac{K_{G}}{\varepsilon^{2}},=-\frac{a+b j}{\varepsilon^{2}} \\
\lambda_{i}\left(\varepsilon e^{0}\right)=\frac{K_{G}}{\varepsilon^{2}}=\frac{a+b j}{\varepsilon^{2}}, \\
\lambda_{i}\left(\varepsilon e^{j \pi / 2}\right)=-\frac{K_{G}}{\varepsilon^{2}}=-\frac{a+b j}{\varepsilon^{2}} .
\end{gathered}
$$

Again, using (25)-(27), $C_{0}$ can be calculated depending on $K_{G}=a+b j$. If $b \neq 0$, as represented in Fig. 3 (i), the $360^{\circ}$ revolution produces a clockwise crossing: $C_{0}=-1$. If $b=0$, $0^{-}$and $0^{+}$both fall in the real axis and $C_{0}$ has to be carefully examined. With $a<0$, Fig. 3 (j) shows how $0 \mathrm{~Hz}$ falls in the real positive axis, and $C_{0}=-1$. If $a>0$ two different cases should be distinguished depending on the phase at $0^{+} \mathrm{Hz}$. For $\varphi\left(\lambda_{i}\left(0^{+}\right)\right)<-180^{\circ}$ (Fig. $\left.3(\mathrm{k})\right)$ an additional crossing is obtained and $C_{0}=-2$, while for $\varphi\left(\lambda_{i}\left(0^{+}\right)\right)>-180^{\circ}$ (Fig. 3 (1)) no crossings are produced and $C_{0}=0$.

5) More than two integrators in the eigenvalue $\lambda_{i}(s), k \geq$ 3: With $k \geq 3 \mathrm{a}+180^{\circ} k$ rotation in the clockwise direction is obtained,

$$
\lambda_{i}\left(\varepsilon e^{j \phi}\right)=\frac{K_{G}}{\varepsilon^{k}} e^{-j k \phi}
$$

Evaluated for the same angles of segment $A B$ than before

$$
\lambda_{i}\left(\varepsilon e^{-j \pi / 2}\right)=\frac{K_{G}}{\varepsilon^{k}} j^{k},
$$

$$
\begin{gathered}
\lambda_{i}\left(\varepsilon e^{0}\right)=\frac{K_{G}}{\varepsilon^{k}}, \\
\lambda_{i}\left(\varepsilon e^{j \pi / 2}\right)=\frac{K_{G}}{\varepsilon^{k}}(-j)^{k} .
\end{gathered}
$$

From these equations, following the same reasoning procedure as before, the cases with a greater number of integrators are a repetition of the ones already analyzed, changing the number of complete revolutions. It should be noticed that the table provided in [24] for SISO systems, matches Table I if $b=0$, meaning that the transfer function under analysis has no complex valued coefficients.

$C_{0}$ has been determined in this section for each eigenvalue. The parameter $C_{0}$ in (14) will be the sum of the crossings at $0 \mathrm{~Hz}$ obtained in all the eigenvalues.

\section{APPLICATION EXAMPLES AND EXPERIMENTAL RESULTS}

The MIMO generalized Bode Criterion is based on the analysis of the Bode diagram, thus, it becomes strongly interesting to design appropriate control loops for power electronic systems. In this section, the proposed stability criterion is used to illustrate the design of controllers for two typical applications in power electronics.

\section{A. Three-phase grid-connected power converter}

The first system under study is the one represented in Fig. 5, a three-phase power converter connected to the grid through an $L C L$ filter. The converter current $i_{c o n v}$, can be typically controlled in the $d q$ frame to achieve zero tracking error of the fundamental frequency component by means of a simple PI controller. Nevertheless, cross-coupling terms appear between both $d q$ axis, and the system has to be modeled as a MIMO system to properly represent its dynamics [14].

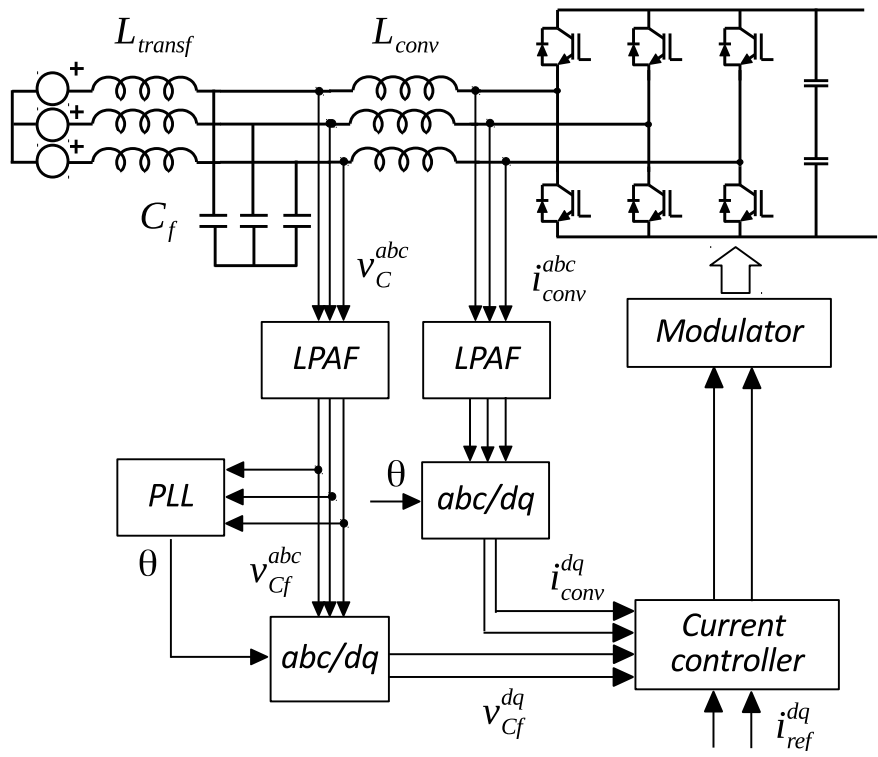

Fig. 5. System under analysis. 
In Fig. 5 the converter current control in the $d q$ frame has been represented. The $L C L$ filter is formed by the converter inductance $L_{c o n v}$, the filter capacitor, $C_{f}$, and the transformer leakage inductance, $L_{\text {transf }}$. The filter capacitor voltage and the converter current measurements are filtered by a low pass analog filter, LPAF. The PLL, receiving the capacitor voltage filtered measurements provides the angle for the transformation to $d q$. The filtered variables are transformed to the synchronous reference frame $(d q)$. Each current component is controlled by a PI controller. At the output of the controller, the capacitor voltage is added, in order to improve grid disturbances rejection. The required action in the SRF is transformed back to the stationary reference frame and the modulator determines the switching orders for every individual switch. This general overview of the control loop is useful to identify all the elements. Nevertheless, to properly study the system stability, a detailed model of each of these elements is required.

As it can be seen, some elements within the control loop, such as the PI controller and capacitor voltage positive feedback are defined in the synchronous reference frame, or $d q$ axis, while others, such as the $L C L$ filter, LPAF and the existing delays within the control loop are defined in the stationary reference frame. In this paper, all the elements are transformed to $d q$ to analyze the system stability and design the controller.

LPAF is a first order low pass filter, $\operatorname{LPAF}(s)=1 /\left(\tau_{l p} s+\right.$ $1)$, applied to all the measurements. This filter can be easily expressed in $d q$ axis applying the transformation presented in [31], which leads to

$$
\left[L P A F^{d q}(s)\right]=\frac{1}{2}\left[\begin{array}{cc}
\operatorname{LPAF}_{1}(s) & \operatorname{LPAF}_{2}(s) \\
-L P A F_{2}(s) & L P A F_{1}(s)
\end{array}\right]
$$

where $L P A F_{1}(s)=L P A F\left(s+j \omega_{0}\right)+L P A F\left(s-j \omega_{0}\right)$ and $L P A F_{2}(s)=j L P A F\left(s+j \omega_{0}\right)-j L P A F\left(s-j \omega_{0}\right)$. It is clear from (32) that the low pass analog filter expressed in the synchronous reference frame has cross-coupling terms.

The delay of one sample time, introduced by the computation in the DSP, and the zero order hold, that represents the application of the control action during a sample time, are modeled by means of a fourth order Pade approximation derived in [32]. In the following, it will be denoted by $D_{\text {conv }}(s)$, which has also to be expressed in $d q$ coordinates, obtaining a matrix $\left[D_{\text {conv }}^{d q}(s)\right]$, with the same symmetry as (32). Its diagonal elements are equal to $D_{\text {conv } 1}(s)=D_{\text {conv }}(s+$ $\left.j \omega_{0}\right)+D_{\text {conv }}\left(s-j \omega_{0}\right)$, and its antidiagonal terms equal to $D_{\text {conv } 2}(s)=j D_{\text {conv }}\left(s+j \omega_{0}\right)-j D_{\text {conv }}\left(s-j \omega_{0}\right)$.

The last element that needs to be expressed in the synchronous reference frame is the $L C L$ filter. The typical $L C L$ plant transfer function is also expressed in $d q$ using the transformation in [31], obtaining a six order transfer function matrix [17], $\left[\operatorname{Plant}(s)^{d q}\right]$, with the same symmetry as (32). The components of matrices $\left[L P A F^{d q}(s)\right],\left[D_{\text {conv }}^{d q}(s)\right]$ and $\left[\operatorname{Plant}(s)^{d q}\right]$ can be easily computed using a dedicated software such as Matlab, however, their expression are too long to be reproduced in here.
The PI controller in Fig 6 is already defined in $d q$ and it has the following expression

$$
\left[P I^{d q}(s)\right]=\left[\begin{array}{cc}
K_{p} \frac{T_{n} s+1}{T_{n} s} & 0 \\
0 & K_{p} \frac{T_{n} s+1}{T_{n} s}
\end{array}\right],
$$

where $T_{n}$ and $K_{p}$ are the integral time constant and the proportional gain.

With all the elements within the control loop defined and expressed in $d q$, the control loop diagram can be represented in Fig. 6. There are two plants defined in this figure, $\left[\operatorname{Plant}_{v v}^{d q}(s)\right]$ correlates the converter voltage to the capacitor filter voltage, while $\left[\operatorname{Plant}_{v i}^{d q}(s)\right]$ correlates the converter voltage to the converter side current. It should be noted that all the elements defined in Fig. 6 are symmetric matrices. Consequently, the open loop transfer matrix, $[H(s)]$, is also symmetric,

$$
\left\{\begin{array}{l}
I_{\text {conv } f}^{d}(s) \\
I_{\text {conv } f}^{q}(s)
\end{array}\right\}=\left[\begin{array}{cc}
H_{1}(s) & H_{2}(s) \\
-H_{2}(s) & H_{1}(s)
\end{array}\right]\left\{\begin{array}{l}
\varepsilon^{d}(s) \\
\varepsilon^{q}(s)
\end{array}\right\},
$$

and its eigenvalues are $\lambda_{1,2}(s)=H_{1}(s) \pm j H_{2}(s)$. The system parameters are summarized in Table II.

Each eigenvalue has an integrator, introduced by the PI controller. The DC-gain without integrators, $K_{G}$, of each eigenvalue is:

$$
\begin{aligned}
& \text { - } K_{G}^{\lambda_{1}}=-0.1469+1.2132 j \\
& \text { - } K_{G}^{\lambda_{2}}=-0.1469-1.2132 j
\end{aligned}
$$

According to Table I, if $k=1$ and $K_{G}$ has a negative real part $(a<0)$, the eigenvalue has a negative crossing. Each eigenvalue has a negative crossing, so $C_{0}=-2$.

There are no unstable poles in $[H(s)]$, regardless of the proportional gain, so $P=0$ in both control loops.

According to the MIMO Generalized Bode Criterion

$$
Z=P-\left[2\left(C^{+}-C^{-}\right)+C_{0}\right],
$$

with no open-loop right-half plane poles, $P=0$, the closedloop system is stable, i.e. $Z=0$, if

$$
-2\left(C^{+}-C^{-}\right)=C_{0}
$$

In this way, the net sum of $\left(C^{+}-C^{-}\right)$has to be equal to 1. For a $T_{n}$ equal to $30 \mathrm{~ms}$ and a $K_{p}$ equal to 1 , the eigenvalues Bode diagram is represented in Fig. 7 (a). It has been proven in Section III that the set of eigenvalues is defined only by

TABLE II

SYSTEM PARAMETERS.

\begin{tabular}{c|c|c}
\hline \hline Parameter & Symbol & Value \\
\hline Converter inductance & $L_{\text {conv }}$ & $2.2 \mathrm{mH}$ \\
Converter inductance series resistance & $R_{\text {conv } s}$ & $100 \mathrm{~m} \Omega$ \\
Grid side inductance & $L_{\text {transf }}$ & $1 \mathrm{mH}$ \\
Grid side inductance series resistance & $R_{g t s}$ & $70 \mathrm{~m} \Omega$ \\
Filter capacitor & $C$ & $10 \mu \mathrm{F}$ \\
Damping resistor & $R_{d}$ & $6 \Omega$ \\
Sampling period & $T_{\text {samp }}$ & $298.6 \mu \mathrm{s}$ \\
Analog filter time constant & $\tau_{l p}$ & $100 \mu \mathrm{s}$ \\
\hline \hline
\end{tabular}




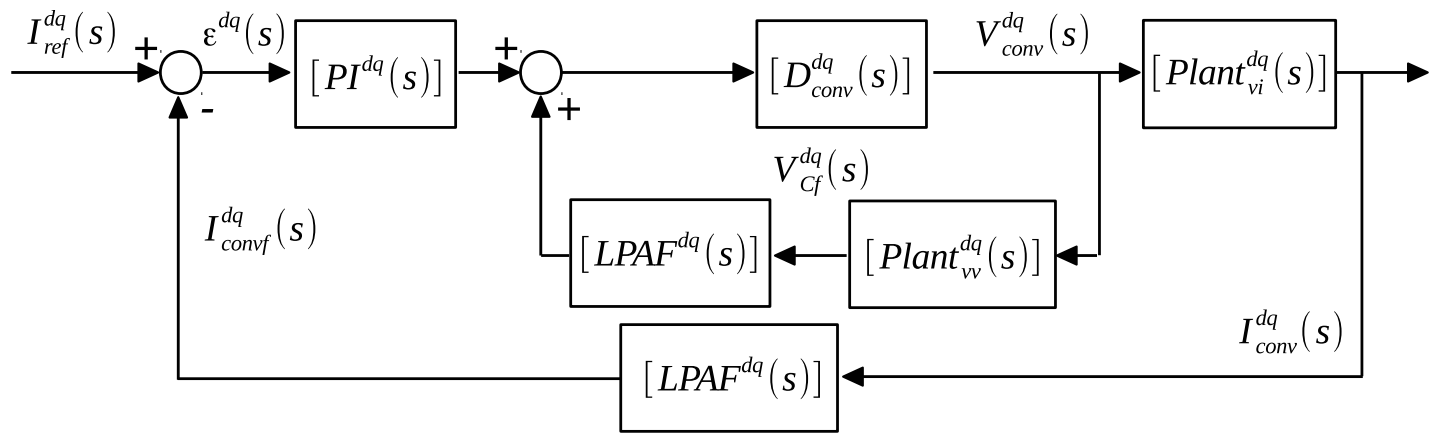

Fig. 6. Control loop diagram.

its positive frequency response, so the stability limits can be easily obtained by inspection of Fig. 7 (a). The minimum proportional gain, $K_{p}$ min , can be determined from the gain margin at the first phase cross-over frequency. To guarantee the $C^{+}$crossing, $K_{p}$ has to be greater than $K_{p}$ min , equal to 0.104 for this application, otherwise $C^{+}-C^{-}=0$, and the closed loop system will have two unstable poles. However, there is an additional stability limit determined by the maximum proportional gain, $K_{p \max }$. This limit can be obtained by examining the gain margin at the second phase cross-over frequency, in this example $K_{p \max }=8$. If the proportional gain is increased over $K_{p \max }$, a $C^{-}$crossing is introduced that compensates the $C^{+}$crossing, so $C^{+}-C^{-}=0$, and again, the closed loop system will have two unstable poles.

An intuitive range of stability has been derived from the eigenvalues' Bode diagram. Obtaining the same stability range by inspection of the Nyquist diagram in Fig. 7 (b) becomes more complicated. $K_{p} \min$ is the required gain to avoid $(-1, \mathrm{j} 0)$ encirclement of $\lambda_{1,2}$. In Fig. 7 (b), for the system under study, $K_{p \text { min }}$ can be intuitively determined from the distance to $(-1, j 0)$ of the two eigenvalues Nyquist diagrams. However, the value for $K_{p} \max$ cannot be seen in such a straightforward way. In contrast to the Bode diagram, the Nyquist diagram is not represented in logarithmic scale, so when $\omega$ tends to infinity, determining the gain margins at the phase cross-over frequencies become more complicated. This is the case even for a simple system such as the one under study. $K_{p} \max$ is the gain that introduces a clockwise encirclement in the Nyquist diagram of $\lambda_{1}$ and it has been marked in Fig. 7 (b). Comparing Fig. 7 (a) and (b) it becomes clear that the Bode diagram presents a more intuitive design approach. Moreover, the crossings at $0 \mathrm{~Hz}$ cannot be determined from the Nyquist diagram and should be computed by knowing that the case under study is an example of the one represented in Fig. 3 (f). It should also be noted that specialized softwares, such as Matlab, plot the Nyquist diagram at positive frequencies and assumes symmetry for the negative frequencies. For this reason, a customized Matlab function needs to be programmed to draw Fig. 7 (b) and properly represent the positive and negative frequencies.

The converter current step response is examined through simulation and represented in Fig. 8 for two different proportional gains. In Fig. 8 (a) $K p$ is equal to 1.122 , greater than $K_{P} \min$ and lower than $K_{P} \max$, so the system is stable, as predicted by the application of the MIMO Generalized Bode Criterion. In contrast, in Fig. 8 (b), $K p$ has been reduced to 0.03 , lower than $K_{P} \min$. Consequently, the system has two RHP poles, showing an unstable step response.
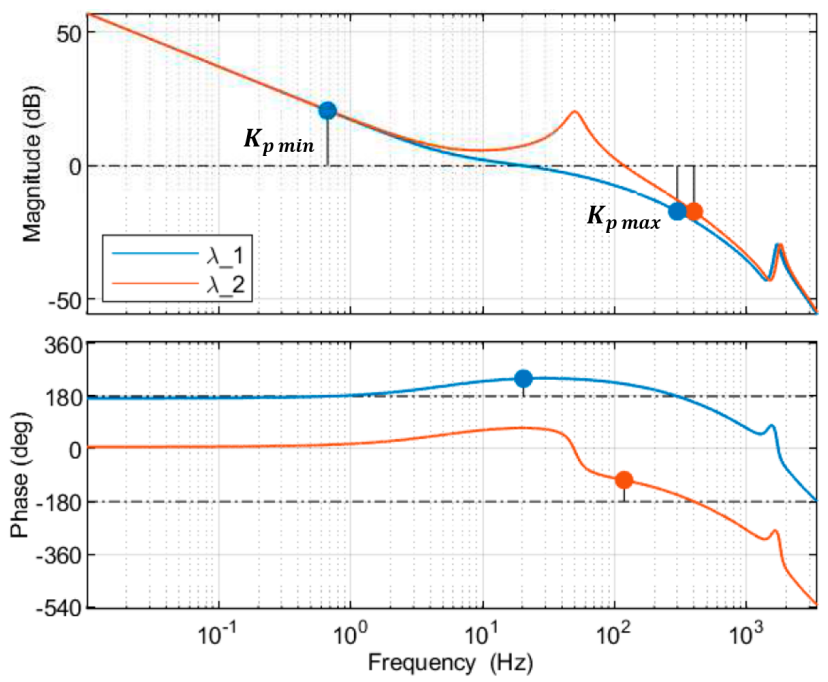

(a)

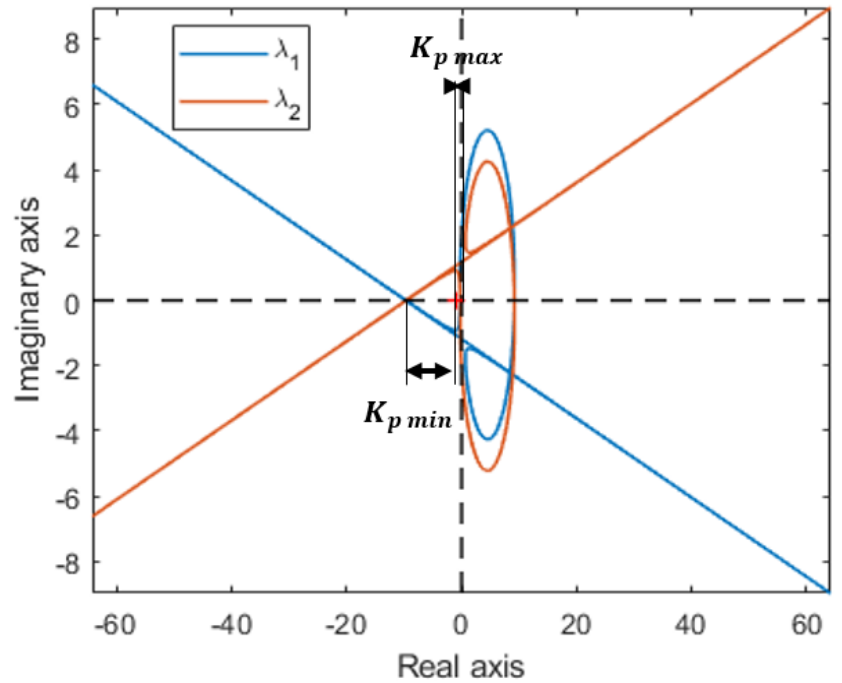

(b)

Fig. 7. Bode diagrams of the eigenvalues for $K_{p}=1$ and $T_{n}=30 \mathrm{~ms}$ (a) and Nyquist diagram of the eigenvalues for the same parameters (b). 


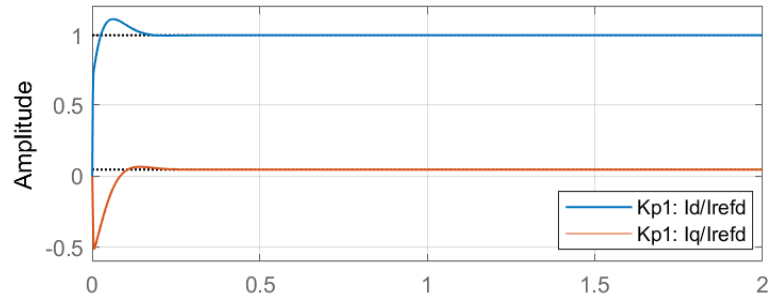

(a)

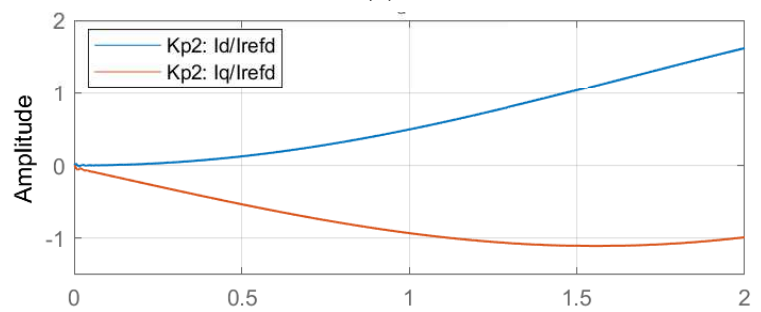

(b)

Fig. 8. Closed-loop poles for $K_{p} \min <K_{p}<K_{p} \max$ (a) and for $K_{p}<$ $K_{p} \min (\mathrm{b})$.

This theory has also been tested in the three-phase power converter shown in Fig. 9, whose parameters are the ones provided in Table II. This converter is controlled by means of an Arduino Due in $d q$ axis using the strategy described in this section. In Fig. 10, a step is introduced in the $d$ axis (in blue), with the controller gain set equal to $K_{p}=0.03$. Initially, the system is unstable $\left(K_{p}<K_{p \text { min }}\right)$, and the converter current in both $d$ axis (green curve) and $q$ axis (in pink) start to grow, as it happened in the simulation results of Fig. 8 (b). However, if the proportional gain is suddenly changed to 1.122 $\left(K_{p \min }<K_{p}<K_{p \max }\right)$, the system becomes stable and rapidly recovers from instability.

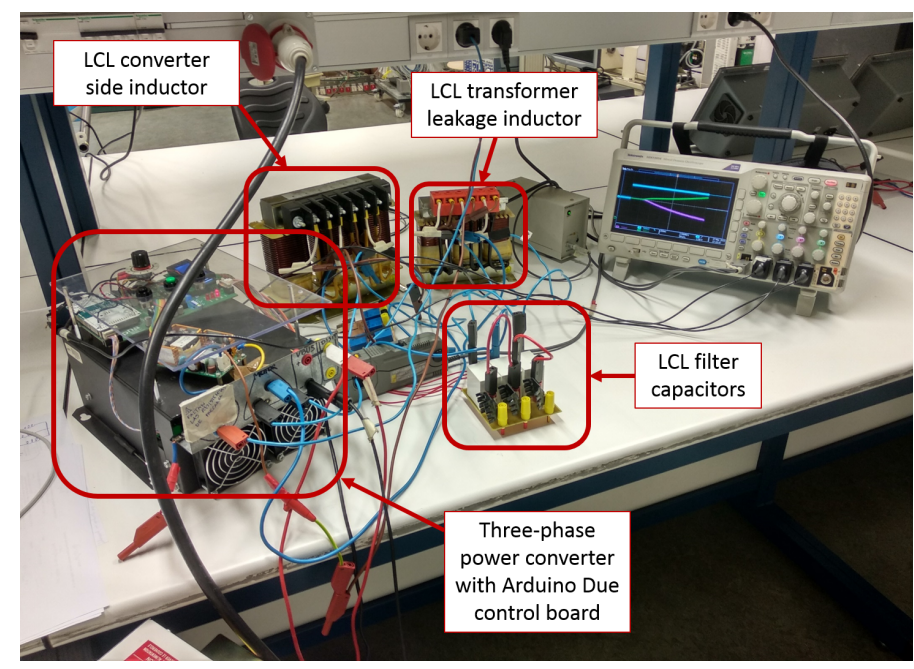

Fig. 9. Test-bench used for the experimental results.

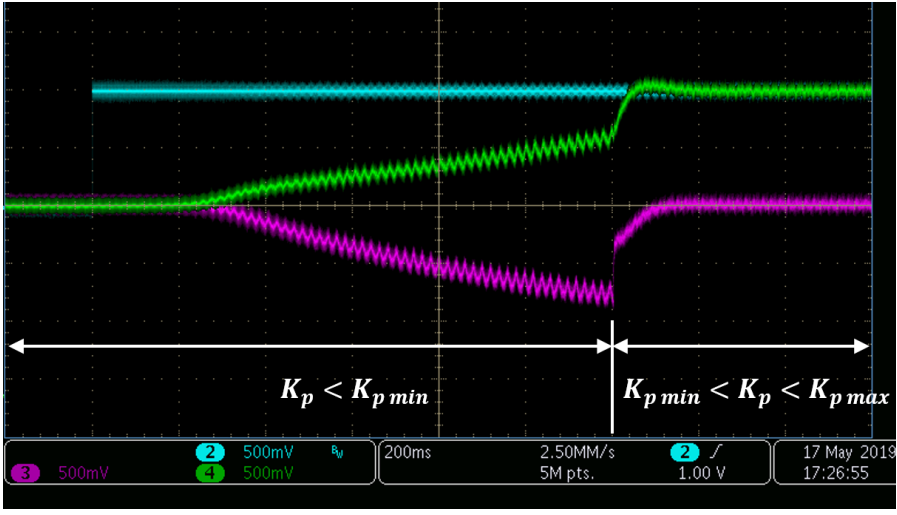

Fig. 10. Step response transiently changing the controller gain from $K_{p}<$ $K_{p \min }$ (unstable) to $K_{p \min }<K_{p}<K_{p \max }$ (stable).

\section{B. Two different power converters connected in parallel to the grid}

Fig. 11 represents the second system under analysis, two three-phase power converters connected in parallel to the grid. Each power converter has the same topology as the one represented in Fig. 5, using the same control loop structure in $d q$ axis. However, as indicated in Fig. 11 by the superscripts $I$ and $I I$, the output filter components and controller parameters can be different. Consequently, the stability analysis and controller design cannot be made by the aggregation of both power converters [33].

The first approach to design the controller of each power converter is to treat them as independent systems. This is realized in many applications, where different manufacturers build their converters and synthesize their controllers without any knowledge of the rest of power converters. Following this design option, two independent control diagrams, as the ones shown in Fig. 6, have to be analyzed. To properly study the system stability, the low pass analog filters, $L P A F^{I}$ and $L P A F^{I I}$, the delay of one sample time and zero order hold, are translated into $d q$ axis, as it is done in the previous example. Analogously, the $L C L$ output filter is also modeled in $d q$. The converter parameters are summarized in Table III, where the filter components are expressed in per-unit values (p.u.). Two independent eigenvalues are computed for each power converter, $\lambda_{1,2}^{I}$ for converter I and $\lambda_{1,2}^{I I}$ for converter II.

To determine the existence of $C_{0}$ crossings, the DC-gain without integrators, $K_{G}$, of each eigenvalue is examined:

- $K_{G}^{\lambda_{1}^{I}}=-0.2131+1.0686 j$

TABLE III

SYSTEM PARAMETERS FOR CONVERTER $I$ AND CONVERTER $I I$.

\begin{tabular}{c|c|c|c|c}
\hline \hline Parameter & Symbol $I$ & Value $I$ & Symbol $I I$ & Value $I I$ \\
\hline Converter inductance & $L_{\text {conv }}^{I}$ & 0.08 p.u. & $L_{\text {conv }}^{I I}$ & 0.05 p.u. \\
\hline Filter capacitor & $C_{f}^{I}$ & 0.05 p.u. & $C_{f}^{I I}$ & 0.095 p.u. \\
\hline Damping resistor & $R_{d}^{I}$ & $1.8 \Omega$ & $R_{d}^{I I}$ & $2 \Omega$ \\
\hline Analog filter time constant capacitor & $\tau_{l p}^{I}$ & $100 \mu \mathrm{s}$ & $\tau_{l p}^{I I}$ & $100 \mu \mathrm{s}$ \\
\hline Sampling period & $T_{\text {samp }}^{I I}$ & $298.6 \mu \mathrm{s}$ & $T_{\text {samp }}^{I I}$ & $298.6 \mu \mathrm{s}$ \\
\hline Grid inductance & $L_{\text {transf }}$ & 0.03 p.u. & $L_{\text {transf }}$ & 0.03 p.u. \\
\hline Grid voltage & $V_{g}$ & $230 \mathrm{~V}$ & $V_{g}$ & $230 \mathrm{~V}$ \\
\hline Converter rated power & $S_{r}^{I}$ & $14.2 \mathrm{~kW}$ & $S_{r}^{I I}$ & $14.2 \mathrm{~kW}$ \\
\hline \hline
\end{tabular}




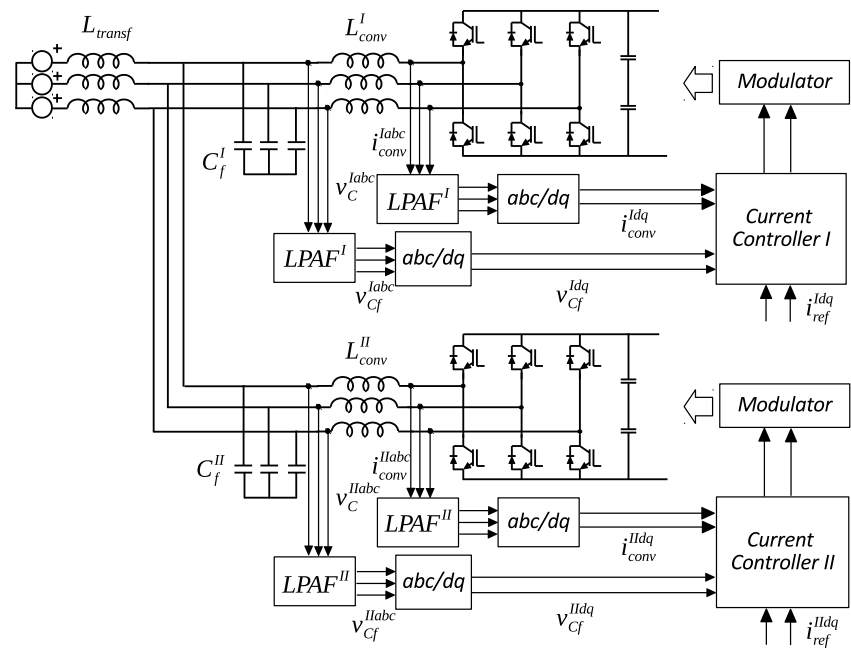

Fig. 11. System under analysis: two three-phase different power converters connected in parallel to the grid.

- $K_{G}^{\lambda_{2}^{I}}=-0.2131-1.0686 j$

- $K_{G_{I I}^{\lambda_{1} I}}^{\lambda_{1}}=-0.1507+1.6665 j$

- $K_{G}^{\lambda_{2}^{I I}}=-0.1507-1.6665 j$

Each eigenvalue has an integrator $(k=1)$, introduced by the PI controller. According to Table I, as the real part of each eigenvalue is negative $(a<0)$, each eigenvalue has a negative crossing at the origin. Consequently, $C_{0}$ can be calculated in both control loops:

- Converter $I: C_{0}^{I}=-2$

- Converter II: $C_{0}^{I I}=-2$

None of the open-loop transfer matrices has unstable poles, i.e. $P^{I}=0$ and $P^{I I}=0$. Thus, according to (36) the closedloop systems are stable, i.e. $Z^{I}=0$ and $Z^{I I}=0$, if $2\left(C^{+}-\right.$ $C^{-}$) is equal to 2 in both control loops. Fig. 12 (a) shows the eigenvalues' Bode diagram if both control loops are analyzed independently. The blue curves are converter's I eigenvalues, while the red ones are those of converter II. This Bode diagram has been represented for a PI controller with a $T_{n}^{I}$ equal to $0.019 \mathrm{~ms}$ and a $K_{p}^{I}=1$ in converter $I$, while in converter $I I$ $T_{n}^{I I}=0.0064$ and $K_{p}^{I I}=1$.

An intuitive range of stability can be found for both control loops so that in each of them the required $C^{+}$crossing is guaranteed. As it can be deduced from Fig. 12 (a), if $0.1161<$ $K_{p}^{I}<11.37$ converter $I$ is stable, while if $0.0551<K_{p}^{I I}<$ 9.45 converter $I I$ is stable as well. In order to achieve a fast dynamic response, $K_{p}^{I}=K_{p}^{I I}=7$ is selected.

A simulation has been performed using Matlab SimPowerSystems library to test the current control loops' adjustment and the results are shown in Fig. 12 (b). Initially, only converter $I$ is connected, it can be seen from the grid current waveforms that the system is stable. At time instant $0.6 \mathrm{~s}$ converter $I I$ is connected in parallel to converter $I$ and the system becomes unstable, as both power converters interact. However, when converter $I$ is disconnected at $0.7 \mathrm{~s}$, the system rapidly becomes stable again. This simulation proves that the stability analysis and the controller adjustment made is correct. If only one converter is connected to the grid, it shows a fast
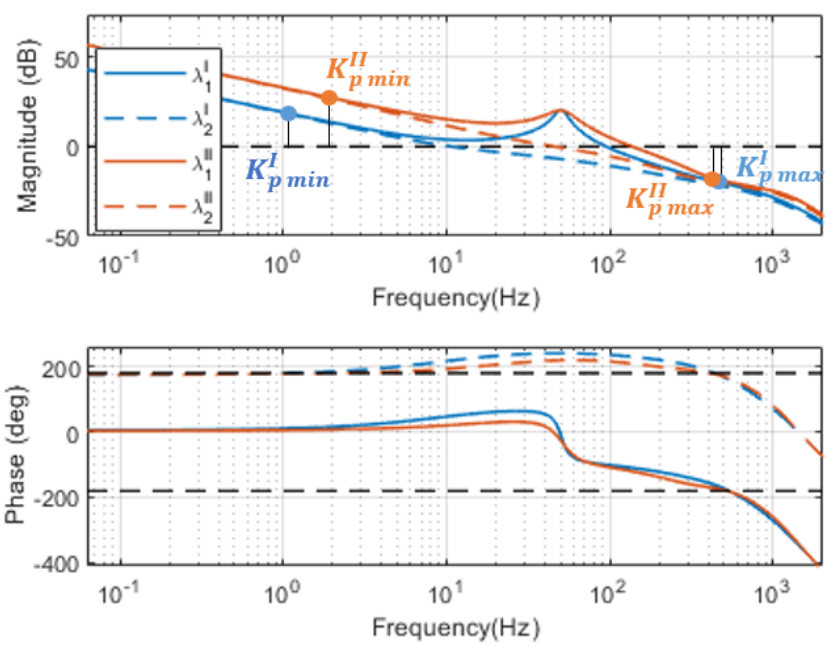

(a)

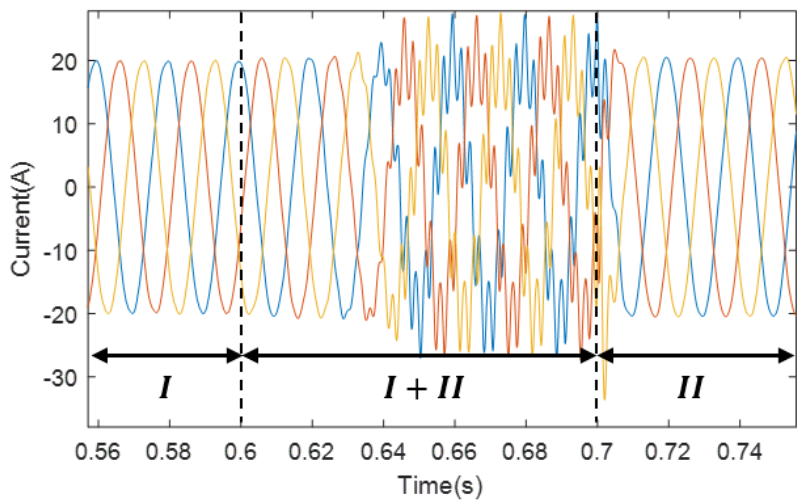

(b)

Fig. 12. Eigenvalues' Bode diagram considering both control loops independent (a) and grid-current transiently connecting and disconnecting converter $I$ and $I I(\mathrm{~b})$.

and stable dynamic response. However, as both current control loops are treated independently, the instability detected when both of them are connected in parallel is neglected in the design procedure.

This limitation in the design procedure can be overcome if both power converters are modeled as a single system instead of considering them independent. From Fig. 11, it becomes intuitive that the voltage imposed by converter $I$ affects converter's II current, and vice versa. These crosscouplings can be modeled using the modeling procedure presented in the previous example. First, the plant is obtained in the stationary reference frame, and then it is translated to $d q$ axis. The following matrix is obtained

$$
\left\{\begin{array}{l}
I_{\text {conv }}^{I d q}(s) \\
I_{\text {conv }}^{I I d q}(s)
\end{array}\right\}=\left[\begin{array}{c|c}
{\left[G_{I}^{d q}(s)\right]} & {\left[G_{I-I I}^{d q}(s)\right]} \\
\hline\left[G_{I I-I}^{d q}(s)\right] & {\left[G_{I I}^{d q}(s)\right]}
\end{array}\right]\left\{\begin{array}{c}
V_{\text {conv }}^{I d q}(s) \\
V_{\text {conv }}^{I I d q}(s)
\end{array}\right\},
$$

where each component is a $2 \times 2$ matrix obtained using the transformation presented in [31].

Even though the plant model becomes a $4 \times 4$ MIMO model, each converter current control loop only measures its own capacitor voltages and currents and actuates on their converter 
voltage, as indicated in Fig. 11, so the model for the rest of the system components, such as low-pass analog filters, PI controllers or delay and zero order hold, do not require any modification. The MIMO Generalized Bode Criterion can be applied to any $n \times n$ MIMO system, so in the following it is applied to the controller design of the resulting $4 \times 4$ open-loop transfer matrix.

The analytical expressions for the eigenvalues become complicated as the number of inputs and outputs grow, for this reason, they are numerically computed using Matlab. Four eigenvalues are obtained, $\lambda_{1,2,3,4}$, each of them with an integrator, provided by the PI controller.

In order to determine the total number of $C_{0}$ crossings, the DC-gain is computed, obtaining that every $K_{G}^{\lambda_{i}}=a+b j$, has a negative real component $(a<0)$. Thus, according to Table I, every eigenvalue has a negative crossing and $C_{0}=-4$.

From (36), the closed loop system is stable, i.e. $Z=0$, if the net sum of $\left(C^{+}-C^{-}\right)$is equal to 2 , as there are no unstable poles in the open-loop transfer matrix. The maximum limits for $K_{p}$ are determined by the eigenvalues' Bode diagram of Fig. 13 (a). Two crossings, with $C^{+}=2$ and $C^{-}=0$, are guaranteed if $0.0794<K_{p}<6.6834$.

The instability observed in Fig. 12 (b) when both power
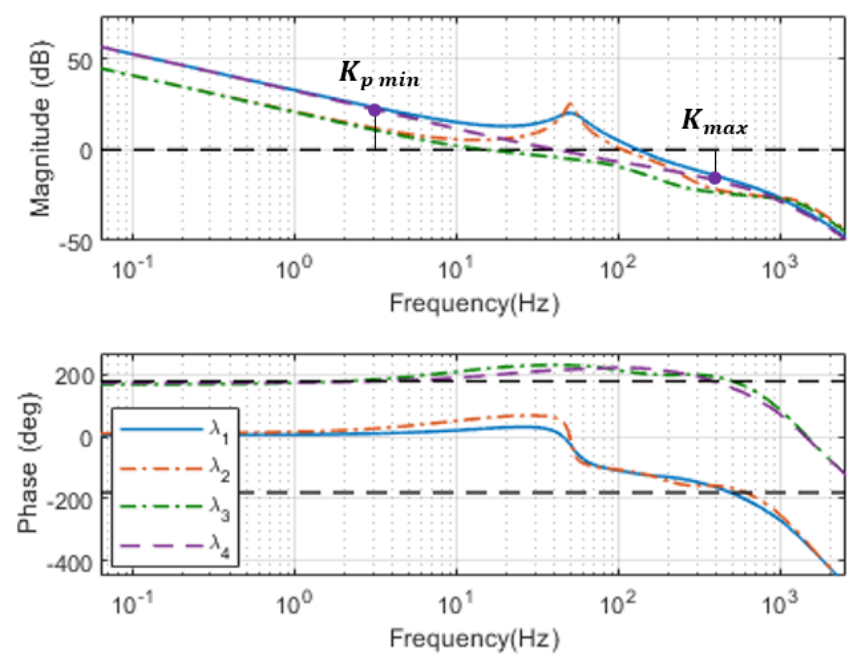

(a)

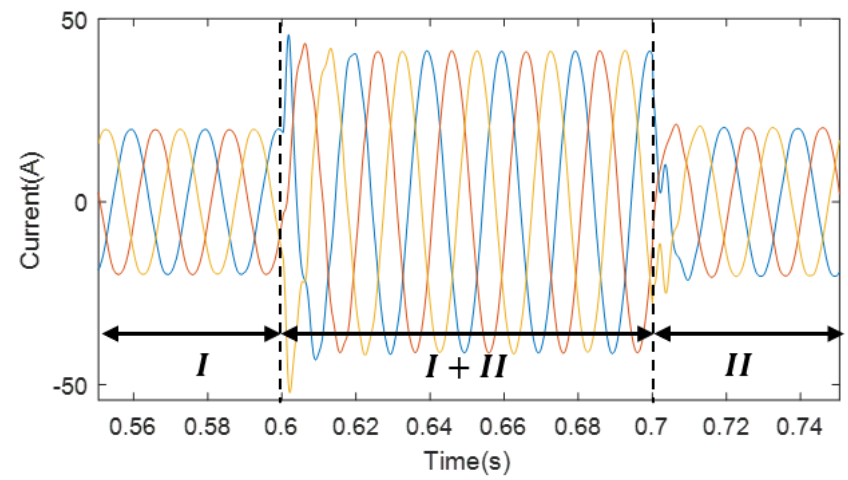

(b)

Fig. 13. Eigenvalues' Bode diagram (a) and grid-current transiently connecting and disconnecting converter $I$ and $I I(b)$. converters are connected in parallel is explained by the new stability limits obtained considering the complete $4 \times 4$ MIMO system. As $K_{p}$ was set to 7 in both power converters, it fell within the instability region, showing the expected unstable behavior with the parallel connection. If $K_{p}^{I}$ and $K_{p}^{I I}$ are set to 5.5 and the simulation is repeated, Fig. 13 (b), the instability is avoided and both converters can operate together and separately.

\section{CONCLUSion}

In this paper the MIMO Generalized Bode Criterion (MIMO-GBC) is proposed. This criterion is based on the Nyquist Generalized Criterion, and can be applied to any MIMO system. It uses the information of the open-loop transfer matrix, but instead of using the eigenvalues' Nyquist diagram, it is based on the Bode diagram, resulting in an intuitive tool for the controller design of power electronic systems. Besides the eigenvalues' frequency response, the MIMO-GBC carefully examines the eigenvalues' response at $0 \mathrm{~Hz}$, as it plays a key role in the system stability.

The proposed stability criterion and controller design approach is applied to the controller adjustment of two typical applications in power electronics. The first one consists in the current control loop of a three-phase power converter, while the second one is the current control loop of two different converters connected in parallel to the grid. In both cases, for $2 \times 2$ and $4 \times 4$ MIMO systems, the MIMO-GBC has proved to be an intuitive tool for the controller design. The validity of the stability criterion has been corroborated through simulation and experimental results.

\section{APPENDIX A}

Let $[H(s)$ be the open-loop transfer matrix of a two-input two-output system

$$
[H(s)]=\left[\begin{array}{cc}
H_{11}(s) & H_{12}(s) \\
H_{21}(s) & H_{22}(s)
\end{array}\right] .
$$

Its two eigenvalues can be computed by solving $\mid \lambda I-$ $H(s) \mid=0$, and have the following expression

$$
\begin{aligned}
\lambda_{1,2}(s)= & \frac{H_{11}(s)+H_{22}(s)}{2} \\
& \pm \sqrt{\left(\frac{H_{11}(s)-H_{22}(s)}{2}\right)^{2}+H_{12}(s) H_{21}(s) .}
\end{aligned}
$$

Each component, $H_{i j}(s)$, has real coefficients, so each individual transfer function satisfies that $H_{i j}(j \omega)=\overline{H_{i j}(-j \omega)}$.

Lets consider $\lambda_{1}(s)$ as the the eigenvalue with the positive square root in (39), so $\lambda_{1}(j \omega)$ is equal to

$$
\begin{aligned}
\lambda_{1}(j \omega)= & \frac{H_{11}(j \omega)+H_{22}(j \omega)}{2} \\
& +\sqrt{\left(\frac{H_{11}(j \omega)-H_{22}(j \omega)}{2}\right)^{2}+H_{12}(j \omega) H_{21}(j \omega)}
\end{aligned}
$$


and it can be rewritten as

$$
\begin{aligned}
& \lambda_{1}(j \omega)=\frac{\overline{H_{11}(-j \omega)}+\overline{H_{22}(-j \omega)}}{2} \\
& +\sqrt{\left(\frac{\overline{H_{11}(-j \omega)}-\overline{H_{22}(-j \omega)}}{2}\right)^{2}+\overline{H_{12}(-j \omega)} \overline{H_{21}(-j \omega)}}
\end{aligned}
$$

Let $z_{1}$ and $z_{2}$ be two complex numbers, the following properties apply:

1) $\overline{z_{1}}+\overline{z_{2}}=\overline{z_{1}+z_{2}}$

2) $\overline{z_{1}} \overline{z_{2}}=\overline{z_{1} z_{2}}$

3) $\left(\overline{z_{1}}\right)^{n}=\overline{\left(z_{1}^{n}\right)}, n$ being an integer number.

If we use the previous properties of complex numbers to (41), it can be expressed as

$$
\begin{aligned}
& \lambda_{1}(j \omega)=\frac{\overline{H_{11}(-j \omega)+H_{22}(-j \omega)}}{2} \\
& +\sqrt{\frac{\left(\frac{H_{11}(-j \omega)-H_{22}(-j \omega)}{2}\right)^{2}+H_{12}(-j \omega) H_{21}(-j \omega)}{2}}
\end{aligned}
$$

If $z_{1}$ has a modulus $\left|z_{1}\right|$ and an argument $\varphi_{1}$ :

- $\underline{\sqrt{z_{1}}}=\sqrt{\left|z_{1}\right|} /-\varphi_{1} / 2+l \pi, l=0,1$

- $\overline{\sqrt{z_{1}}}=\sqrt{\left|z_{1}\right| /-\varphi_{1} / 2-l \pi}, l=0,1$

So for $l=0, \sqrt{z_{1}}=\sqrt{z_{1}}$, while for $l=1, \sqrt{\overline{z_{1}}}=-\overline{\sqrt{z_{1}}}$.

The application of this property to (42) leads to the following two equalities

$$
\lambda_{1}(j \omega)=\overline{\lambda_{1}(-j \omega)} \text { and } \lambda_{2}(j \omega)=\overline{\lambda_{2}(-j \omega)}
$$

and

$$
\lambda_{1}(j \omega)=\overline{\lambda_{2}(-j \omega)} \text { and } \lambda_{2}(j \omega)=\overline{\lambda_{1}(-j \omega)} .
$$

Meaning that in one case each eigenvalue evaluated at positive frequencies is equal to the conjugate of the same eigenvalue evaluated at negative frequencies ((43) with $l=0)$. While in the other case ((44) with $l=1$ ) each eigenvalue evaluated at positive frequencies is equal to the conjugate of the other eigenvalue evaluated at negative frequencies. To illustrate this idea we are going to analyze two examples. First of all, lets consider a symmetric system

$$
[H(s)]=\left[\begin{array}{cc}
H_{1}(s) & H_{2}(s) \\
-H_{2}(s) & H_{1}(s)
\end{array}\right] .
$$

Its eigenvalues are equal to $\lambda_{1,2}(s)=H_{1}(s) \pm j H_{2}(s)$.

Example Al: If $H_{1}(s)=1 / s$ and $H_{2}(s)=0$, the two eigenvalues are equal $\left(\lambda_{1}=\lambda_{2}=1 / s\right)$. Then

$$
\lambda_{1}(j \omega)=\frac{1}{j \omega}
$$

and

$$
\lambda_{1}(-j \omega)=-\frac{1}{j \omega}
$$

So it can be concluded that for this case under consideration, (43) $(l=0)$ applies.
Example A2: If $H_{1}(s)=2 / s$ and $H_{2}(s)=1 / s$, the two eigenvalues are

$$
\begin{aligned}
& \lambda_{1}(s)=\frac{2}{s}+j \frac{1}{s} \\
& \lambda_{2}(s)=\frac{2}{s}-j \frac{1}{s}
\end{aligned}
$$

so,

$$
\begin{aligned}
& \lambda_{1}(j \omega)=\frac{2}{j \omega}+\frac{1}{\omega} \text { and } \lambda_{1}(-j \omega)=-\frac{2}{j \omega}-\frac{1}{\omega} \\
& \lambda_{2}(j \omega)=\frac{2}{j \omega}-\frac{1}{\omega} \text { and } \lambda_{2}(-j \omega)=-\frac{2}{j \omega}+\frac{1}{\omega} .
\end{aligned}
$$

And consequently, (44) $(l=1)$ applies in this case.

In both cases, the set of eigenvalues form closed symmetric curves and only $\pm m 180$ degrees crossings with positive magnitude ( $m$ odd integer) need to be counted at positive frequencies to determine the total number of $C^{+}$and $C^{-}$ crossings.

\section{REFERENCES}

[1] F. Briz, M. W. Degner, and R. D. Lorenz, "Analysis and design of current regulators using complex vectors," IEEE Transactions on Industry Applications, vol. 36, no. 3, pp. 817-825, May 2000.

[2] J. Shen, S. Schröder, H. Stagge, and R. W. De Doncker, "Precise modeling and analysis of dq-frame current controller for high power converters with low pulse ratio," in 2012 IEEE Energy Conversion Congress and Exposition (ECCE), Sep. 2012, pp. 61-68.

[3] J. Yim, S. Sul, B. Bae, N. R. Patel, and S. Hiti, "Modified current control schemes for high-performance permanent-magnet ac drives with low sampling to operating frequency ratio," IEEE Transactions on Industry Applications, vol. 45, no. 2, pp. 763-771, March 2009.

[4] S. Zhou, J. Liu, L. Zhou, and Y. Zhang, "Dq current control of voltage source converters with a decoupling method based on preprocessed reference current feed-forward," IEEE Transactions on Power Electronics, vol. 32, no. 11, pp. 8904-8921, Nov 2017.

[5] J. Dannehl, C. Wessels, and F. W. Fuchs, "Limitations of voltageoriented pi current control of grid-connected pwm rectifiers with $l c l$ filters," IEEE transactions on industrial electronics, vol. 56, no. 2, pp. 380-388, 2008.

[6] A. G. Yepes, F. D. Freijedo, . Lopez, and J. Doval-Gandoy, "Analysis and design of resonant current controllers for voltage-source converters by means of nyquist diagrams and sensitivity function," IEEE Transactions on Industrial Electronics, vol. 58, no. 11, pp. 5231-5250, Nov 2011.

[7] A. Vidal, F. D. Freijedo, A. G. Yepes, J. Malvar, . López, and J. DovalGandoy, "Transient response evaluation of stationary-frame resonant current controllers for grid-connected applications," IET Power Electronics, vol. 7, no. 7, pp. 1714-1724, July 2014.

[8] F. Blaabjerg, R. Teodorescu, M. Liserre, and A. V. Timbus, "Overview of control and grid synchronization for distributed power generation systems," IEEE Transactions on Industrial Electronics, vol. 53, no. 5, pp. 1398-1409, Oct 2006.

[9] A. Timbus, M. Liserre, R. Teodorescu, P. Rodriguez, and F. Blaabjerg, "Evaluation of current controllers for distributed power generation systems," IEEE Transactions on Power Electronics, vol. 24, no. 3, pp. 654-664, March 2009.

[10] L. Harnefors, X. Wang, A. G. Yepes, and F. Blaabjerg, "Passivitybased stability assessment of grid-connected vscs-an overview," IEEE Journal of emerging and selected topics in Power Electronics, vol. 4, no. 1, pp. 116-125, 2015.

[11] M. Ramezani, S. Li, and S. Golestan, "Analysis and controller design for stand-alone vsis in synchronous reference frame," IET Power Electronics, vol. 10, no. 9, pp. 1003-1012, 2017.

[12] L. Sainz, M. Cheah-Mane, L. Monjo, J. Liang, and O. Gomis-Bellmunt, "Positive-net-damping stability criterion in grid-connected vsc systems," IEEE Journal of Emerging and Selected Topics in Power Electronics, vol. 5, no. 4, pp. 1499-1512, 2017. 
[13] A. Rygg, M. Molinas, C. Zhang, and X. Cai, "On the equivalence and impact on stability of impedance modeling of power electronic converters in different domains," IEEE Journal of Emerging and Selected Topics in Power Electronics, vol. 5, no. 4, pp. 1444-1454, Dec 2017.

[14] L. Harnefors, "Modeling of three-phase dynamic systems using complex transfer functions and transfer matrices," IEEE Transactions on Industrial Electronics, vol. 54, no. 4, pp. 2239-2248, Aug 2007.

[15] E. Prieto-Araujo, A. Junyent-Ferré, D. Lavèrnia-Ferrer, and O. GomisBellmunt, "Decentralized control of a nine-phase permanent magnet generator for offshore wind turbines," IEEE Transactions on Energy Conversion, vol. 30, no. 3, pp. 1103-1112, Sep. 2015.

[16] I. Zoric, M. Jones, and E. Levi, "Arbitrary power sharing among threephase winding sets of multiphase machines," IEEE Transactions on Industrial Electronics, vol. 65, no. 2, pp. 1128-1139, Feb 2018.

[17] J. Samanes, A. Urtasun, E. Gubia, and A. Petri, "Robust multisampled capacitor voltage active damping for grid-connected power converters," International Journal of Electrical Power \& Energy Systems, vol. 105, pp. 741-752, 2019

[18] S. Sastry and M. Bodson, Adaptive control: stability, convergence and robustness. Courier Corporation, 2011.

[19] X. Wang, F. Blaabjerg, and P. C. Loh, "Virtual rc damping of lc1filtered voltage source converters with extended selective harmonic compensation," IEEE Transactions on Power Electronics, vol. 30, no. 9, pp. 4726-4737, 2015.

[20] B. Wen, D. Dong, D. Boroyevich, R. Burgos, P. Mattavelli, and Z. Shen, "Impedance-based analysis of grid-synchronization stability for threephase paralleled converters," IEEE Transactions on Power Electronics, vol. 31, no. 1, pp. 26-38, Jan 2016.

[21] X. Li, J. Fang, Y. Tang, X. Wu, and Y. Geng, "Capacitor-voltage feedforward with full delay compensation to improve weak grids adaptability of lcl-filtered grid-connected converters for distributed generation systems," IEEE Transactions on Power Electronics, vol. 33, no. 1, pp. 749-764, Jan 2018.

[22] H. W. Bode, "Relations between attenuation and phase in feedback amplifier design," The Bell System Technical Journal, vol. 19, no. 3, pp. $421-454,1940$

[23] J. Hahn, T. Edison, and T. F. Edgar, "A note on stability analysis using bode plots," Chemical Engineering Education, vol. 35, no. 3, pp. 208211, 2001.

[24] D. Lumbreras, E. L. Barrios, A. Urtasun, A. Ursua, L. Marroyo, and P. Sanchis, "On the stability of advanced power electronic converters: the generalized bode criterion," IEEE Transactions on Power Electronics, 2018.

[25] L. Harnefors and H.-P. Nee, "Model-based current control of ac machines using the internal model control method," IEEE Transactions on Industry Applications, vol. 34, no. 1, pp. 133-141, 1998.

[26] F. B. Del Blanco, M. W. Degner, and R. D. Lorenz, "Dynamic analysis of current regulators for ac motors using complex vectors," IEEE Transactions on Industry Applications, vol. 35, no. 6, pp. 1424-1432, 1999.

[27] J. Rodriguez, M. P. Kazmierkowski, J. R. Espinoza, P. Zanchetta H. Abu-Rub, H. A. Young, and C. A. Rojas, "State of the art of finite control set model predictive control in power electronics," IEEE Transactions on Industrial Informatics, vol. 9, no. 2, pp. 1003-1016, 2012.

[28] J. M. Maciejowski, "Multivariable feedback design," Wokingham, England: Addison-Wesley, 1989.

[29] M. Vidyasagar, R. Bertschmann, and C. Sallaberger, "Some simplifications of the graphical nyquist criterion," IEEE transactions on automatic control, vol. 33, no. 3, pp. 301-305, 1988

[30] S. Skogestad and I. Postlethwaite, Multivariable feedback control: analysis and design. Wiley New York, 2007, vol. 2.

[31] D. N. Zmood, D. G. Holmes, and G. H. Bode, "Frequency-domain analysis of three-phase linear current regulators," IEEE Transactions on Industry Applications, vol. 37, no. 2, pp. 601-610, March 2001.

[32] J. Samanes and E. Gubía, "Sensorless active damping strategy for parallel interleaved voltage source power converters with lcl filter," in 2017 IEEE Applied Power Electronics Conference and Exposition (APEC), March 2017, pp. 3632-3639.

[33] J. L. Agorreta, M. Borrega, J. López, and L. Marroyo, "Modeling and control of $n$-paralleled grid-connected inverters with lcl filter coupled due to grid impedance in pv plants," IEEE Transactions on Power Electronics, vol. 26, no. 3, pp. 770-785, March 2011



Javier Samanes (S'15-M'18) was born in Pamplona, Spain, in 1990. He received the M.Sc. degree in electrical engineering from the Public University of Navarre (UPNA), Pamplona, Spain, in 2014 and the M.Sc. degree in renewable energy engineering in 2016 from the same institution. He obtained the $\mathrm{Ph} . \mathrm{D}$. degree in electrical engineering from the UPNA in 2018

In 2014, he joined the Electrical Engineering, Power Electronics and Renewable Energy Research Group (INGEPER) at the UPNA, where he is currently Assistant Professor and member of the Institute of Smart Cities (ISC). In 2018 he was visiting scholar in the Center for Power Electronic Systems (CPES) at Virginia Tech, USA. His research interests include power electronics and renewable energies.

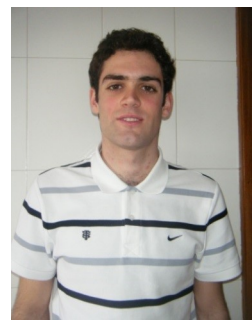

Andoni Urtasun (S'11-M'16) was born in Pamplona, Spain, in 1987. He received the M.Sc. degree in electrical engineering from the Public University of Navarre (UPNA), Pamplona, Spain, and from the Institut National Polytechnique de Toulouse, Toulouse, France, both in 2010, and the Ph.D. degree in electrical engineering from the UPNA in 2015.

In 2010, he joined the Electrical Engineering, Power Electronics and Renewable Energy research group, UPNA, where he is currently Associate Professor. He has been involved in more than 15 research projects, mainly in cooperation with industry, and coauthor more than 30 papers in international journals and conferences. His research interests include control of power electronics and renewable energies.

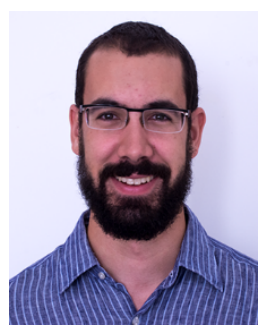

Ernesto barrios (S'12) was born in Pamplona, Spain, in 1988. He received the B.Sc. and M.Sc. degree in electrical engineering in 2009 and 2012, respectively, and the Ph.D. degree in electrical engineering in 2015, all from the Public University of Navarra, Spain.

In 2011, he joined the Electrical Engineering, Power Electronics and Renewable Energy research group (INGEPER), Public University of Navarre, where he is currently an associate professor and researcher. During 6 months in 2016, he was an academic guest in the Power Electronic Systems Laboratory in ETH Zurich, Switzerland. His main research interests include high frequency magnetics, wide bandgap power semiconductor devices and power converters for renewable energies, particularly for photovoltaics.

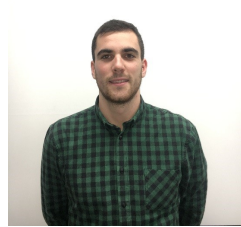

David Lumbreras (S'18) was born in Pamplona, Spain, in 1993. He received both the B.Sc. and M.Sc. degree in Electrical Engineering in 2015 and 2017, respectively, from the Public University of Navarre, Pamplona. He did his Master Thesis at the Technische Universität Braunschweig (Germany) on the topic of advanced control for power electronics.

In 2015, he joined the Electrical Engineering, Power Electronics and Renewable Energy Research Group, from the Public University of Navarre, where he is currently working toward the Ph.D. degree. His research interests include power electronics, design of power converters and renewable energies. 


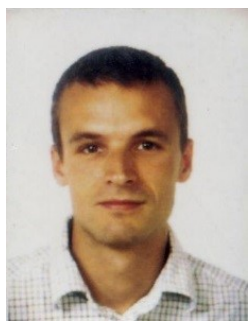

Jesús López (M’05) was born in Pamplona, Spain, in 1975. He received the M.Sc. degree in industrial engineering from the Public University of Navarra, Pamplona, Spain, in 2000. In 2008 he received the $\mathrm{Ph} . \mathrm{D}$. degree in industrial engineering from the Public University of Navarra, Spain, in collaboration with the LAPLACE laboratory, Toulouse, France.

In 2001, he joined the Power Electronic Group, Electrical and Electronic Department at the Public University of Navarra, where he is currently an Assistant Professor and is also involved in research projects mainly in co-operation with industry. His research interests are in the field of power electronics, power systems quality and renewable energies, such as wind turbines and photovoltaic plants.

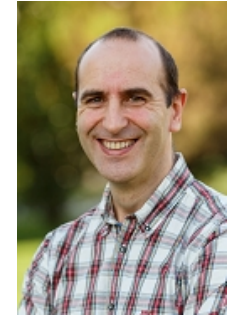

Eugenio Gubia received the M.Sc. and Ph.D. degrees in industrial engineering from the Public University of Navarre, Spain, in 1995 and 2003, respectively.

He joined the Electrical and Electronic Department of the Public University of Navarre in 1996, where he is currently an associate professor and member of the Institute of Smart Cities (ISC). In 2002, he joined the Electrical Engineering, Power Electronics, and Renewable Energy Research Group (INGEPER). From June to December 2005, he worked as a guest researcher at the Center for Power Electronics Systems (CPES) in the field of electromagnetic compatibility. His research interests are in the field of power electronics, renewable energy systems, high-frequency phenomena, and electromagnetic compatibility.

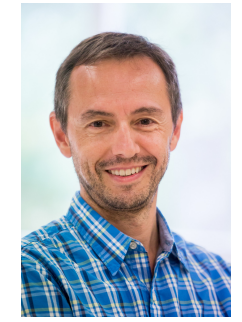

Pablo Sanchis received the M.Sc. degree in Electrical Engineering, the M.Sc. degree in Management and Business Administration, and the Ph.D. degree in Electrical Engineering from the Public University of Navarre (UPNA, Pamplona, Spain) in 1994, 1995, and 2002, respectively.

From 1996 to 1998, he was a Guest Researcher at Delft University of Technology, The Netherlands.

In 1998, he joined the Department of Electrical, Electronic and Communications Engineering of the Public University of Navarre, where he is currently Associate Professor. He is also Director of the UPNA Chair for Renewable Energies and Head of the Research Group in Electrical Engineering, Power Electronics, and Renewable Energies. Previously, he was Vice Dean of the School of Industrial and Telecommunications Engineering and Director of the University Research Resources and Structures Unit.

$\mathrm{He}$ has been involved in more than 80 research projects both with public funding and in co-operation with industry and is the coinventor of eight patents. He has also coauthored more than 140 papers and contributions in international journals and conferences, and supervised ten Ph.D. thesis. His research interests include renewable energies, power electronics, electric energy storage technologies, grid integration of renewable energies, and electric microgrids.

Dr. Sanchis received the UPNA Research Award for the Best Technical Paper, in 2013, and the UPNA Excellence in Teaching Award, in 2017. 